\title{
Ginsenoside Rg1 alleviates repeated alcohol exposure-induced psychomotor and cognitive deficits
}

\author{
Lu Huang ${ }^{1,2+}$, Zhuang Peng ${ }^{1,5+}$, Cong Lu ${ }^{3}$, Ying Chen ${ }^{4}$, Jing-wei Lv ${ }^{3}$, Meng Qin ${ }^{5}$, Duan-fang Liao ${ }^{1 *}$, \\ Xin-min Liu ${ }^{1,3^{*}}$ and Zhe Shi ${ }^{1^{*}}$
}

\begin{abstract}
Background: Chronic alcohol consumption disrupts psychomotor and cognitive functions, most of which are subserved by the dysfunction of hippocampus. Dysregulated excitatory glutamatergic transmission is implicated in repeated alcohol induced psychomotor and cognitive impairment. Ginsenoside Rg1, one of the main active ingredient of the traditional tonic medicine Panax ginseng C.A. Meyer (Araliaceae), has been used to treat cognitive deficits. Particularly, Rg1 has been demonstrated to improve hippocampus-dependent learning in mice and attenuate glutamate-induced excitotoxicity in vitro. Thus, in the present research, we sought to investigate the therapeutic effects of Ginsenoside Rg1 on repeated alcohol induced psychomotor and cognitive deficits in hippocampal-dependent behavioral tasks and unravel the underpinnings of its neuroprotection.
\end{abstract}

Methods: Male ICR (CD-1) mice were consecutively intragastrically treated with 20\% (w/v) alcohol for 21 days. Then, behavior tests were conducted to evaluate repeated alcohol induced psychomotor and cognitive deficits. Histopathological changes, and biochemical and molecular alterations were assessed to determine the potential neuroprotective mechanism of Rg1.

Results: The results suggested that $\mathrm{Rg} 1$, at the optimal dose of $6 \mathrm{mg} / \mathrm{kg}$, has the potential to ameliorate repeated alcohol induced cognitive deficits by regulating activities of NR2B containing NMDARs and excitotoxic signaling.

Conclusion: Our findings further provided a new strategy to treat chronic alcohol exposure induced adverse consequences.

Keywords: Ginsenoside Rg1, Repeated alcohol exposure, Psychomotor and cognitive deficits, Excitatory glutamatergic transmission, NR2B containing NMDARs

\section{Background}

Alcohol-use disorders are among the most disabling disease categories for the global burden of disease [1, 2]. In humans, chronic alcohol consumption results

*Correspondence: dfliao@hnucm.edu.cn; liuxinmin@hotmail.com; zhezhe1106@163.com

${ }^{\dagger}$ Lu Huang and Zhuang Peng contributed equally to this article. ${ }^{1}$ Division of Stem Cell Regulation and Application, Key Laboratory for Quality Evaluation of Bulk Herbs of Hunan Province, Hunan University of Chinese Medicine, Changsha 410208, Hunan, China

Full list of author information is available at the end of the article in alcoholism and leads to brain shrinkage and loss of nerve cells at specific brain regions via an excitotoxic and oxidative mechanism, which has been regarded as the main pathogenic factor for neurodegeneration [3-5]. Many individuals diagnosed with alcoholism have been reported to exert measurable chronic cognitive impairment [6-8]. Excessive alcohol consumption disrupts cognitive functions in a battery of behavioral tasks in both clinical and experimental studies [9-11], most of which are subserved by the dysfunction of hippocampus [12, 13]. The hippocampus is critical in encoding

(c) The Author(s) 2020. This article is licensed under a Creative Commons Attribution 4.0 International License, which permits use, sharing, adaptation, distribution and reproduction in any medium or format, as long as you give appropriate credit to the original author(s) and the source, provide a link to the Creative Commons licence, and indicate if changes were made. The images or other third party material in this article are included in the article's Creative Commons licence, unless indicated otherwise in a credit line to the material. If material is not included in the article's Creative Commons licence and your intended use is not permitted by statutory regulation or exceeds the permitted use, you will need to obtain permission directly from the copyright holder. To view a copy of this licence, visit http://creativeco mmons.org/licenses/by/4.0/. The Creative Commons Public Domain Dedication waiver (http://creativecommons.org/publicdomain/ zero/1.0/) applies to the data made available in this article, unless otherwise stated in a credit line to the data. 
diverse features of experiences such as spatial locations, landmarks, visual features of the environment, goal locations, conditioned stimuli, and sequences of events [14]. Hippocampal-dependent cognitions are particularly susceptible to the deleterious effects of chronic alcohol exposure, which can result in aberrant structural and functional changes [15-18].

Chronic alcohol consumption leads to elevated levels of extracellular glutamate and triggers excessive activation of various glutamatergic receptors [19-21]. $\mathrm{N}$-methylD-aspartate receptors (NMDARs) are not only pivotal regulators in normal physiological processes in the central nervous system (CNS), but also important target of alcohol [22]. Chronic exposure to alcohol induces expression and functional alterations of NMDARs [23, 24]. Therefore, regulating the activity of NMDAR signaling could be an effective way to rescue chronic alcohol exposure induced neuronal dysfunction.

Ginsenoside Rg1, one of the main active ingredient of the traditional tonic medicine Panax ginseng C.A. Meyer (Araliaceae), has been used to treat cognitive deficits with neuroprotection, anti-oxidative stress, anti-apoptosis, anti-inflammation and neurotrophic properties [25-29]. Particularly, Rg1 has been demonstrated to improve hippocampus-dependent learning in mice and attenuate glutamate-induced excitotoxicity in vitro [30-32]. Thus, we hypothesized that Ginsenoside Rg1could exert beneficial effects on chronic alcohol exposure induced cognitive deficits.

In the present research, we sought to investigate the therapeutic effects of $\operatorname{Rg} 1$ on repeated alcohol exposure (RAE) induced psychomotor and cognitive deficits in hippocampal-dependent behavioral tasks and unravel the underpinnings of its neuroprotection.

\section{Methods}

\section{Animals}

Eight to ten weeks old male ICR (CD-1) mice were obtained from Vital River (Beijing, China). They were group-housed under controlled environmental conditions $\left(25{ }^{\circ} \mathrm{C}\right.$ and $50-70 \%$ humidity) with food and water ad libitum. All mice were acclimatized to a 12-h light/dark cycle (lights on at 7:00 a.m. and lights off at 7:00 p.m.). The animal experimental procedures were approved by the Animal Ethics Committee of Institute of medicinal plant development (IMPLAD), CAMS \& PUMC and were conducted strictly according to the National Institutes of Health Guide for the Care and Use of Laboratory Animals (NIH Publications No. 8023, revised 1978).

\section{Drugs and treatment schedule}

Mice were assigned to five groups (Control, Alcohol, Rg1-3 mg, Rg1-6 mg and Rg1-12 mg, $\mathrm{n}=12$ each) in a quasi-random manner after a 3 days acclimatization. Ginsenoside Rg1, purchased from Chengdu Herbpurify (Sichuan, China), was daily intragastrically administrated at the dose of $3 \mathrm{mg} / \mathrm{kg}, 6 \mathrm{mg} / \mathrm{kg}$ and $12 \mathrm{mg} / \mathrm{kg}$ with an intragastric tube in the $\operatorname{Rg} 1$ treatmnt groups for 14 days prior to corresponding alcohol treatment and throughout the experiment. Mice in the control and alcohol group respectively received isovolumetric normal saline with an intragastric tube as well. From day 15 , all mice except in the control group were daily intragastrically administrated alcohol $(20 \% \mathrm{w} / \mathrm{v}$ in isotonic saline) at a dose of $3.4 \mathrm{~g} / \mathrm{kg}$ until the end of behavioral tests to mimic repeated alcohol exposure.

\section{Behavioral procedures Locomotor activity}

Thirty minutes after alcohol treatment, each mouse was initially situated at the center of the tank to freely explore the environment for $3 \mathrm{~min}$. An overhead video camera was used to record the movements, and the total distances traveled in the following $10 \mathrm{~min}$ was analyzed by image analyzer software.

\section{Object location recognition (OLR) test}

The OLR test was used to evaluate teh recognition memory, which has been described in detail in our previous research [33]. In brief, during a 3-days habituation period, mice were allowed to explore the environment freely in the arena with no objects presented for $10 \mathrm{~min}$ each day. On the fourth day, mice were initially placed in the arena where presented two copies of novel objects (A1 and A2) and allowed to explore (5 min per trial) freely during the familiarization period. After a 30-min interval, mice returned to the arena for the test trial, during which one of the original objects were moved into new location ('novel') and the other remained in the previous position ('familiar'). Objects and their placement were presented in a counter-bias manner to avoid positional preferences. A video camera was used to record the exploratory behavior. The behavioral changes were analysed and scored by two double-blind sophisticated experimenters. The discrimination index (DI) formula used for scoring the recognition memory of each animal is as follows: $\mathrm{DI}=(\mathrm{TN}-\mathrm{TF}) /(\mathrm{TN}+\mathrm{TF})$. $\mathrm{TN}$, exploration time on the object changed location; TF, exploration time on the object unchanged location. 


\section{Morris water maze (MWM) test}

The MWM test was administered subsequently to evaluate spatial learning and memory. As previously reported in detail [33], the equipment consists of a stainless steel tub which was divided into four equal quadrants. Make sure the water temperature was maintained at $25{ }^{\circ} \mathrm{C}$. A hyaline platform $(6 \mathrm{~cm}$ in diameter and $15 \mathrm{~cm}$ in height) was submerged $1 \mathrm{~cm}$ below the surface and placed in one of the quadrant (e.g. SE). An overhead video camera was used to record the swimming activity and the data was analyzed by image analyzer software. Thirty minutes after drug treatment, mice were subjected to find the submerged platform three trials per day for four consecutive days, with each trial having a ceiling time of $90 \mathrm{~s}$. The escape latency was recorded according to the time for mice to find the submerged platform. In the probing test day, the platform was removed. Each mouse was freed from quadrant (e.g. NW) opposite to which the platform used in (the target quadrant). Every animal was tested only once and the time spent in the target quadrant was recorded until $90 \mathrm{~s}$.

\section{Tissue preparation}

After finishing behavioral tests, mice were anesthetized and transcardially perfused with ice-cold saline immediately and half number of mice in each group were followed by $4 \%$ paraformaldehyde to fix for histological analysis, the rest of the mice brains were quickly removed and placed on ice in order to dissect the hippocampus prepares for total protein extraction.

\section{Histopathology analysis}

Before being dehydrated, the brains were removed and post-fixed overnight in paraformaldehyde, then embedded in paraffin. Brains were cut into $10 \mu \mathrm{m}$ thick sections in the coronal plane and stained with hematoxylin and eosin. The hippocampus CA1, CA3, and DG subregions in brain sections were used to observe pathological alterations.

\section{Western blotting}

After extracting total proteins, the protein concentration was assayed by a BCA protein assay kit. The protein extracts were subjected to $8 \%$ or $12 \%$ SDS-PAGE (CWBIO, China) and transferred onto a polyvinylidene difluoride (PVDF) membranes. The membrane was treated for $1 \mathrm{~h}$ with blocking solution (5\% skim milk in TBST) and incubated at $4{ }^{\circ} \mathrm{C}$ overnight with the primary rabbit monoclonal antibodies respectively (NR1, CST, 1:1000; NR2B, CST, 1:1000; m-calpain, Abcam, 1:500; STEP Antibody (23E5), Novus Biologicals, 1:1000; p-p38, Abcam, 1:500 and $\beta$-actin, CST, 1:2000). The next day, after incubation with secondary goat-anti-rabbit antibody $(1: 1000, \mathrm{CST})$ for $1 \mathrm{~h}$, the enhanced chemiluminescence (ECL) was then utilized to visualize immunoreactive proteins and the signals were quantified by densitometry with a Western blotting detection system (Quantity One, Bio-Rad, USA).

\section{Biochemical analysis}

The concentration of glutamate (Glu) in the hippocampus was determined by an LC-MS/MS method previously described in detail [14]. After weighed the tissues, ice-cold aqueous acid was prepared to homogenize the tissues, then precipitate protein in formic acid. The supernatant was collected after centrifugation and mixed with an internal standard solution $(300 \mu \mathrm{g} / \mathrm{mL}$ DHBA $)$, then collected $50 \mu \mathrm{l}$ of the mixture to analyze by $\mathrm{LC}-$ MS/MS system. Agilent $1200 \mathrm{HPLC}$ system (Palo Alto, CA, USA), an Applied Biosystem 3200 Q-Trap mass spectrometer (Foster City, CA, USA) and an electrospray ionization source constitute LC-MS/MS instrument. The mobile phase consisted of $6 \mathrm{mM}$ ammonium formate in acetonitrile-water (67.5:32.5, $\mathrm{pH}$ 5.50). The detection limit and quantification ranged from 0.96 to $24.48 \mathrm{nmol} / \mathrm{L}$ and 3.42 to $244.82 \mathrm{nmol} / \mathrm{L}$. The quantification of Glu was according to the ratios of the peak areas of the analyte versus the internal standard.

\section{Statistical analysis}

All analyses were performed by SPSS version 23.0 for Mac (IBM, USA). All data were represented as the mean \pm SEM. Data were analyzed with a one-way analysis of variance (one-way ANOVA) or repeated-measures analysis of variance (RM ANOVA) where statistically appropriate. Post hoc multiple pairwise comparisons were made with the LSD comparisons test after ANOVA when significant effects were detected [34]. The analysis results were only presented when a significant difference was observed. Statistical significance was defined as $P<0.05$ [35].

\section{Results}

Rg1 alleviated RAE induced cognitive deficits in hippocampal-dependent behavioral tasks

To determine RAE induced cognitive deficits, mice were subjected to open field test, object location recognition, and morris watermaze test. Open field test was conducted to assess psychomotor responses of mice after alcohol exposure. As shown in Fig. 1b, hyperactivity was observed in mice received repeated alcohol treatment $\left(\mathrm{F}_{4,35}=11.62, P<0.001\right)$. Mice with $\mathrm{Rg} 1-3 \mathrm{mg} / \mathrm{kg}$ $(P<0.01), 6 \mathrm{mg} / \mathrm{kg}(P<0.001)$ and $12 \mathrm{mg} / \mathrm{kg}(P<0.01)$ treatment exerted less psychomotor response to alcohol 
a

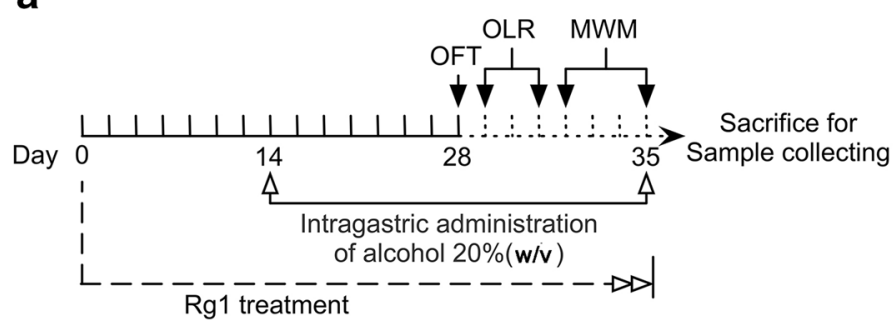

b

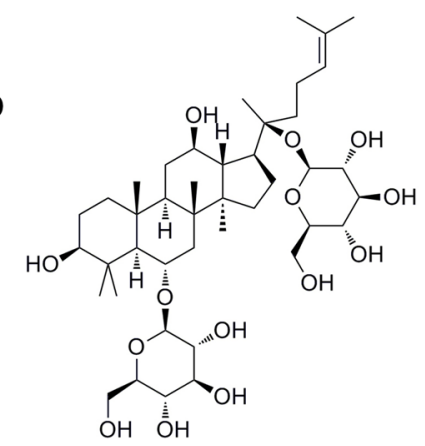

d Sample phase

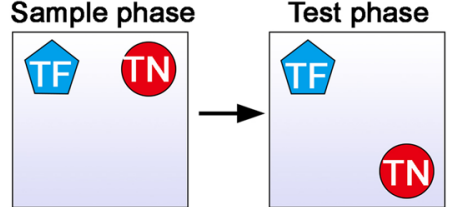

C
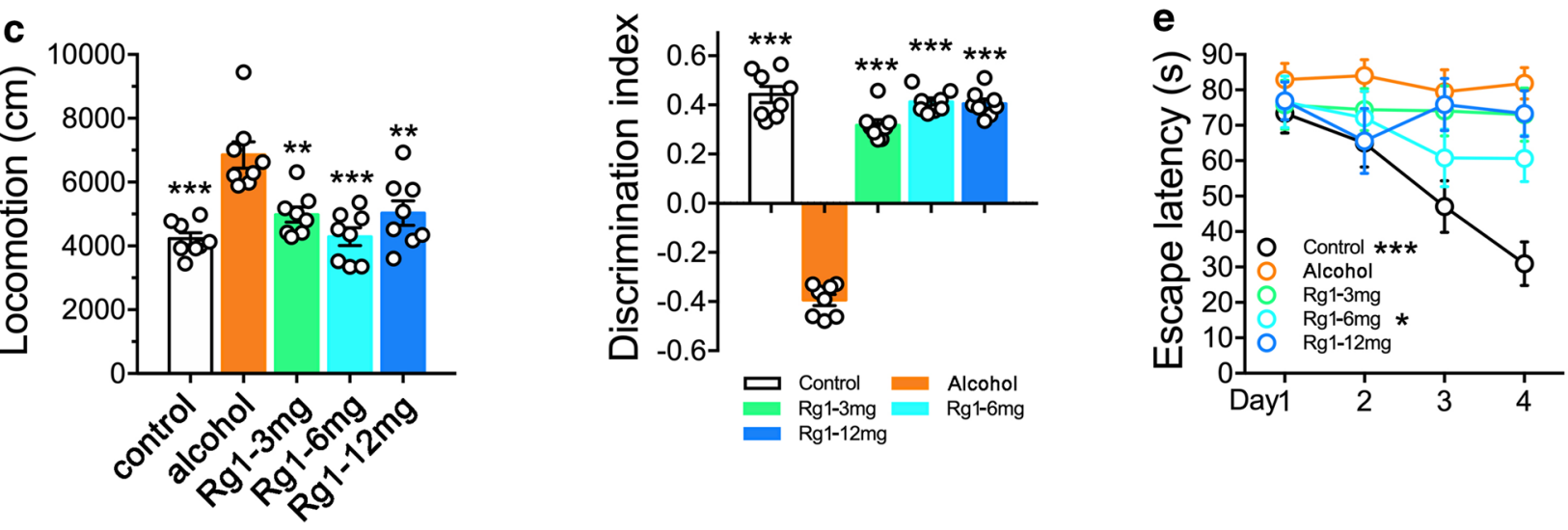

f

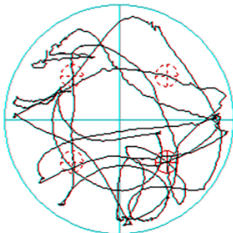

Control

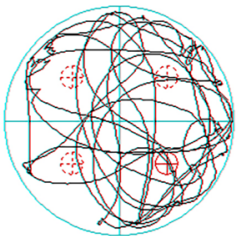

Rg1-3mg

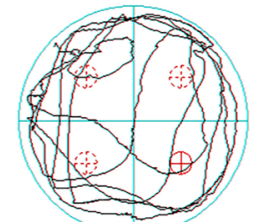

Alcohol

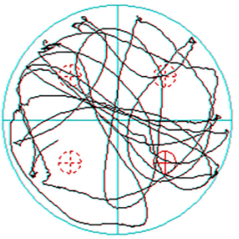

Rg1-6mg
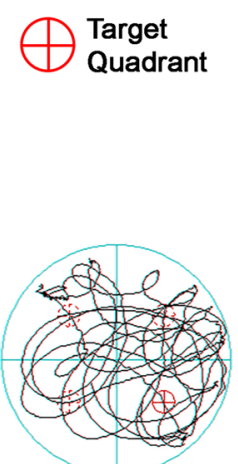

Rg1-12mg

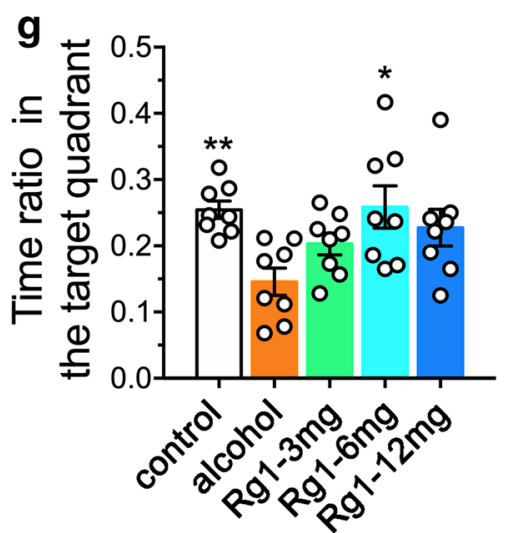

Fig. 1 Ginsenoside Rg1 treatment alleviated repeated alcohol-induced cognitive deficits. a A schematic illustration of the experimental manipulation. $\mathbf{b}$ chemical structure of ginsenoside Rg1. c The open field test showed that Rg1 pretreatment decreased RAE-induced psychomotor response. $\mathbf{d}$ The object location recognition test demonstrated that the impaired recognition ability was recovered after Rg 1 treatment. $\mathbf{e}-\mathbf{g}$ Mice received $\mathrm{Rg} 1$ pretreatment showed better spatial navigation and orientation abilities in the reference learning and memory retention tasks. ( ${ }^{*} P<0.05,{ }^{* *} P<0.01,{ }^{* *} P<0.001$, compared with alcohol group; $\mathrm{n}=8$ per group) 
exposure. Moreover, the object location recognition test was conducted to assess the ability to distinguish both object location and features. We noticed that mice in the alcohol group spent more time to explore the location unchanged object $\left(\mathrm{F}_{4,35}=233.4, P<0.001\right)$, while mice in the $\mathrm{Rg} 1-3 \mathrm{mg} / \mathrm{kg}(P<0.001), 6 \mathrm{mg} / \mathrm{kg}(P<0.001)$ and $12 \mathrm{mg} / \mathrm{kg}(P<0.001)$ treatment groups spent more time to explore the location changed object (see Fig. 1c). Furthermore, the water maze tests were performed to assess the hippocampus-dependent spatial reference memory. As shown in Fig. 1d-f Mice in the alcohol group were obviously retarded to find the invisible platform during the 4 days reference learning task $\left(\mathrm{F}_{4,115}=7.947\right.$, $P<0.001)$ and performed worse to retrieve the spatial memory $\left(\mathrm{F}_{4,35}=4.019, P<0.01\right)$. Notably, mice pretreated with $\mathrm{Rg} 1$ at $6 \mathrm{mg} / \mathrm{kg}$ dosage, exerted better spatial navigation $(P=0.025)$ and orientation $(P=0.0115)$ abilities in the reference learning and memory retention tasks.

\section{Rg1 treatment suppressed RAE-induced} neuro-excitotoxicity in the hippocampus

Then, we determined whether RAE-induced psychomotor and cognitive deficits were associated with pathological alterations in the hippocampus. We conducted
H\&E staining in hippocampal slices. The HE staining could visually show histological changes in neurons. As shown in Fig. 2a-c, in the control mice, neurons in the CA1, CA3 and DG subregions of the hippocampus were round or oval in shape and the nuclei were clear. After RAE, numerous impaired neurons with karyopyknosis, cell gaps, and debris were observed in the CA1, CA3 and DG subregions of the hippocampus in the alcohol group. However, Rg1 treatment significantly reversed RAEinduced morphological alterations in varying degrees. Notably, Rg1 treatment at the dose of $6 \mathrm{mg} / \mathrm{kg}$ showed a better curative effect to protect neurons from alcohol insult.

\section{Rg1 treatment reduced glutamate spillover and NR2B-containing NMDARs activation in the hippocampus}

To determine whether the observed pathological changes in the hippocampus were related to the dysregulation of excitatory glutamatergic transmission. We detected the changes of glutamate content in the hippocampus by a LC-MS/MS method. As shown in Fig. 3a, glutamate content was obviously higher after $\operatorname{RAE}\left(\mathrm{F}_{4,15}=6.756, P=0.037\right) . \operatorname{Rg} 1$ treatment at the

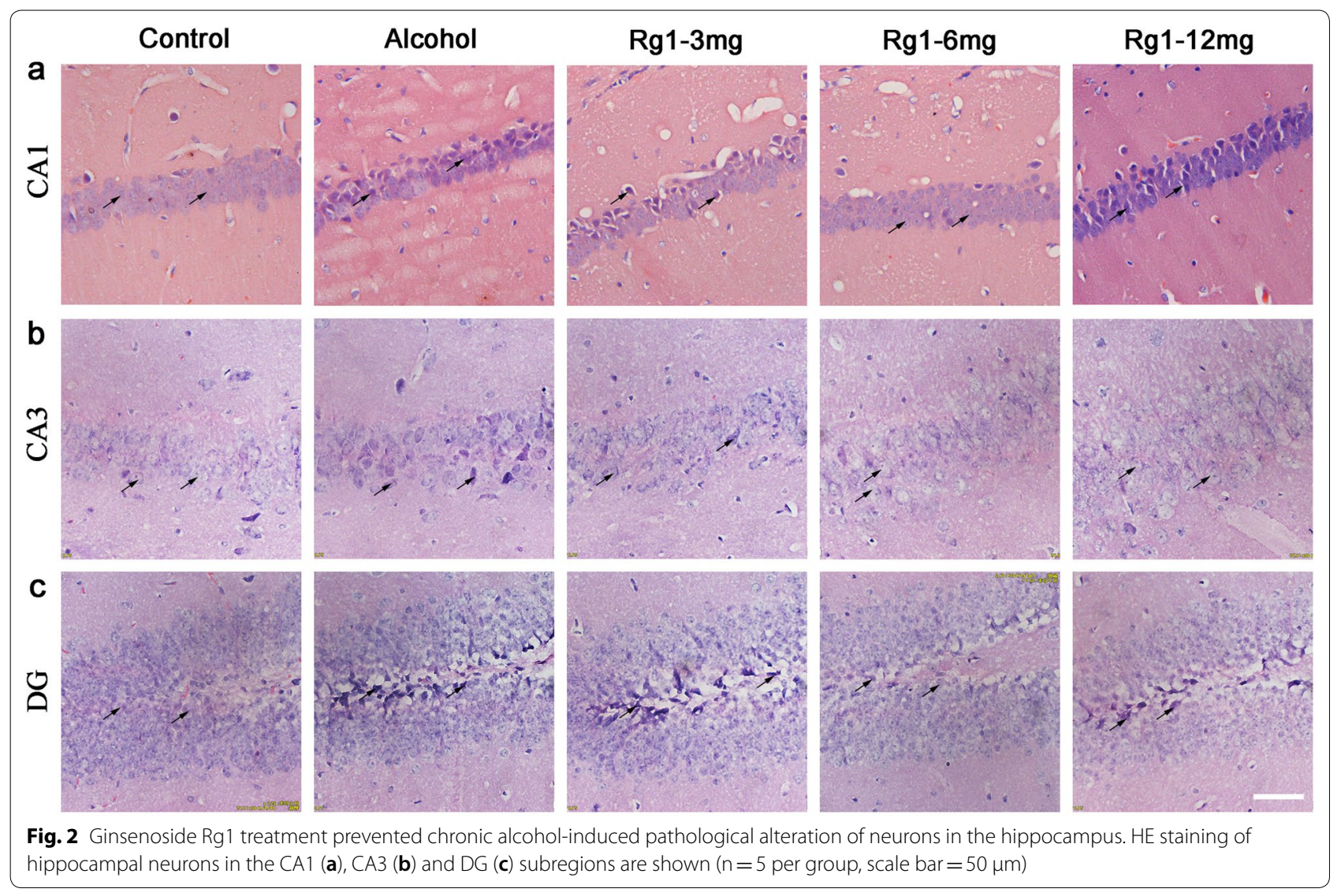



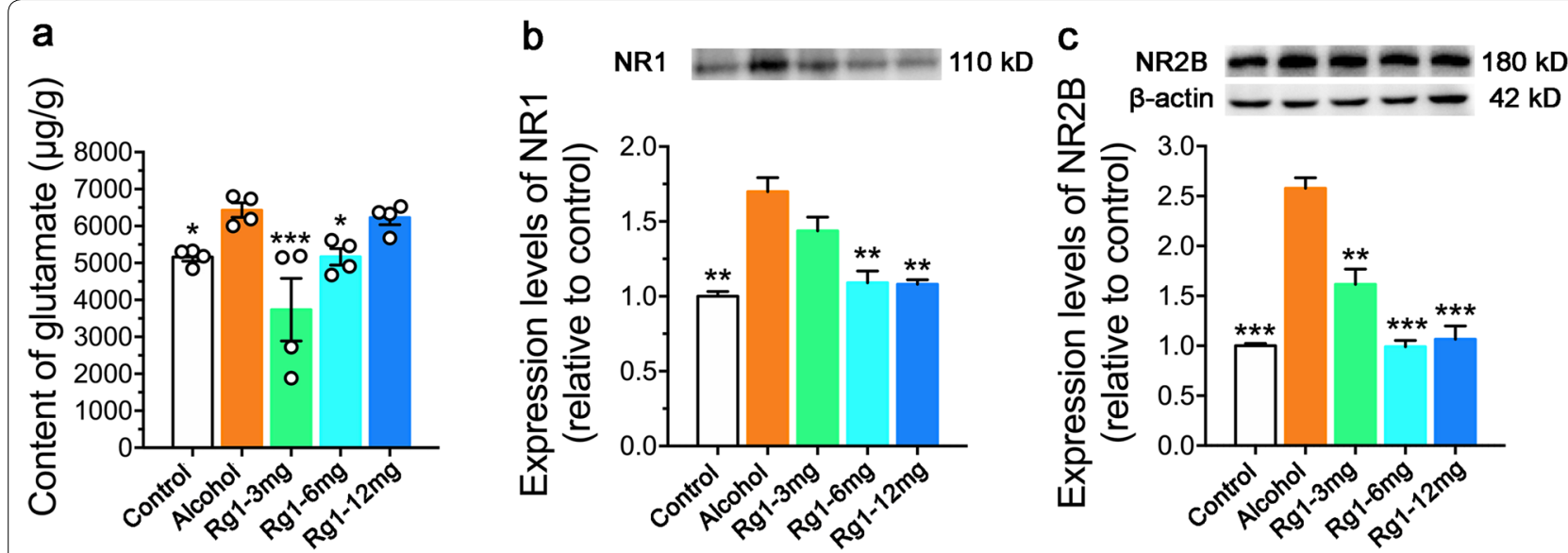

Fig. 3 Ginsenoside Rg1 treatment reduced glutamate spillover and NR2B-containing NMDARs activation in the hippocampus. a Alterations in glutamate content. Expression level changes in NR1 (b) and NR2B (c) in the hippocampus $\left({ }^{*} P<0.05\right.$, ${ }^{* *} P<0.01,{ }^{* *} P<0.001$, compared with alcohol group; $n=4$ per group in glutamate content examination and $n=3$ per group in western blot assay)

doses of $3 \mathrm{mg} / \mathrm{kg}(P<0.001)$ and $6 \mathrm{mg} / \mathrm{kg}(P=0.047)$ significantly reduced glutamate levels in the hippocampus. Moreover, RAE induced excessive glutamate release triggers the activation of NMDARs. We noticed that RAE significantly elevated the expression levels of NR1. Since NR1 is obligatory in the heterotetramer, we further determined the positive correlation between the expression levels of NR1 and NR2B. Thus, it is conceivable RAE could excessively activate NR2B containing NMDARs. Notably, Rg1 treatment inhibited NR2B activation at the dose of $6 \mathrm{mg} / \mathrm{kg}$ and $12 \mathrm{mg} /$ $\mathrm{kg}$. All things considered, Rg1 treatment at the dose of $6 \mathrm{mg} / \mathrm{kg}$ displayed the optimal pharmacological activity in reducing glutamate spillover and inhibiting NR2Bcontaining NMDARs activation.

\section{Rg1 exerts neuroprotective effects via suppressing extrasynaptic NMDARs-mediated excitotoxic signaling}

To reveal the potential mechanism by which Rg1 exerts its neuroprotection, extrasynaptic NMDARs mediated excitotoxic signaling was assessed. As shown in Fig. 4a-d, calpain-2 expression was obviously elevated after RAE. Subsequently, $\mathrm{STEP}_{61}$ converted to $\mathrm{STEP}_{33}$ in repeated alcohol-treated mice. Increased $\mathrm{STEP}_{33}$ expression ultimately activated the phosphorylation of p38 MAPK. Intriguing, Rg1 treatment significantly reversed extrasynaptic NMDARs mediated excitotoxic signaling in a dose-dependent manner. Rg1 treatment suppressed the activation of calpain-2 and the transformation of $\mathrm{STEP}_{61}$ to $\mathrm{STEP}_{33}$, which effectively inhibited the phosphorylation of p38 MAPK. Thus, it can be inferred that Rg1 protected hippocampal neurons by suppressing calpain-2/STEP/p38 excitotoxic cascade.

\section{Discussion}

The consumption pattern of alcohol is critical to determine the nature and extent of pathological and behavioral consequences. Repeated daily administration of alcohol is reliable to induce compulsive alcohol seeking, psychomotor and cognitive deficits [36-38]. Consistently, in the present study, we found that RAE disrupted psychomotor and cognitive functions in several hippocampus-dependent behavioral tasks. Excessive alcohol consumption leads to impairments in behavioral control, learning, memory and executive functions attributed, at least partially, to the integrity of the hippocampus [39-41]. Hippocampus is essential to transmitting circumstantial and contextual information via glutamatergic afferents to the prefrontal cortex and ventral striatum, and retrieves previous experience to guide behavior $[14,42]$.

Chronic alcohol exposure was reported to tolerate to the sedative effects of alcohol and result in withdrawal hyperexcitability [43, 44]. Consistently, as shown in Fig. 1b, locomotor activities of mice were significantly increased after RAE. Rg1 treatment significantly alleviated psychomotor responses to alcohol, which is critical for the development of addiction [45]. Moreover, water maze tests were used to assess spatial reference learning and memory [46]. Hippocampus has been well demonstrated to play a pivotal role in spatial memory $[47,48]$. In the reference learning task, animals are required to find an invisible platform placed in one of four quadrants. They had to learn the location using extrinsic cues and retain this information in the memory retention test. Mice received repeated alcohol administration were obviously unable to find the invisible platform during the 4 days reference learning task and performed worse to retrieve the spatial memory (see Fig. 1d-f). Mice pretreated with 


\section{a}

Calpain-2

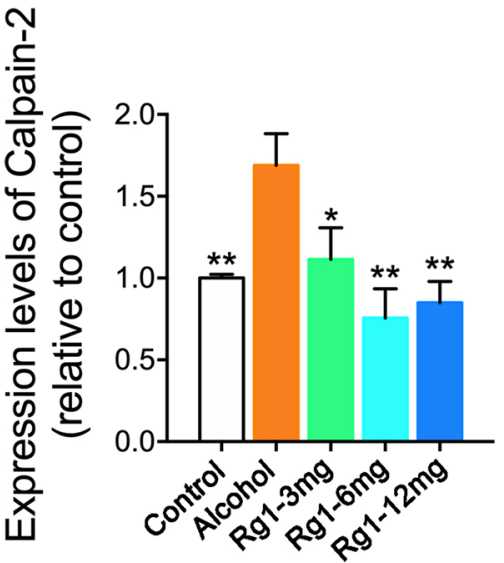

C

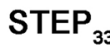

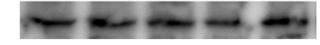

$33 \mathrm{kD}$

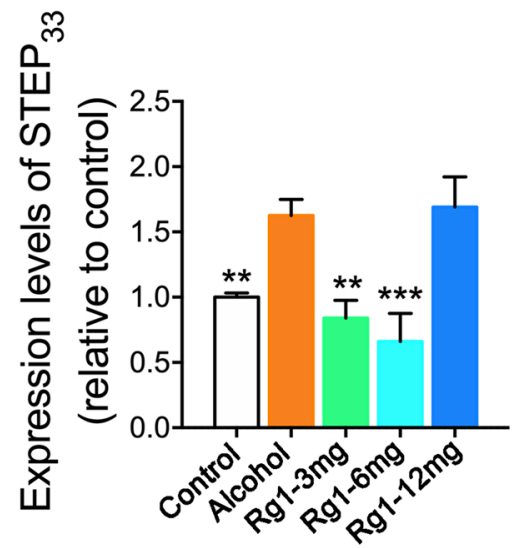

b

STEP $_{61}$

$61 \mathrm{kD}$ d

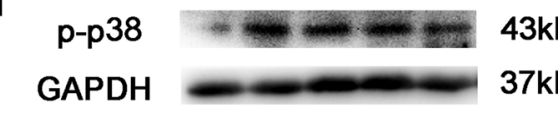

$\infty$

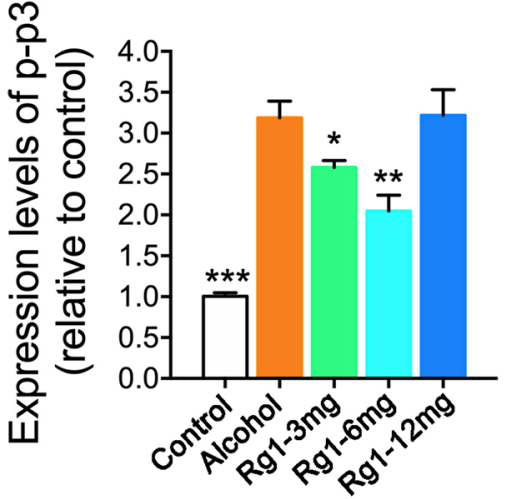

Fig. 4 Ginsenoside Rg1 exerts neuroprotective effects via suppressing extrasynaptic NMDARs-mediated excitotoxic signaling. Expression level changes in calpain-2 (a), STEP61 (b), STEP33 (c) and p-p38 MAPK (d) in hippocampus are shown $\left({ }^{*} P<0.05,{ }^{* *} P<0.01,{ }^{* *} P<0.001\right.$, compared with alcohol group; $n=3$ per group)

$\operatorname{Rg} 1$ (6 mg/kg) exerted better spatial navigation and orientation abilities in the reference learning and memory retention tasks. Furthermore, hippocampus also involves in recognition ability $[49,50]$. The object location recognition task depends on the creature's natural instinct to explore novel items or a novel location [51]. Hippocampal neurons, particularly in the CA1 subregion, are critically involved in encoding both object location and identity information and hence play a key role in forming objectin-place association [52, 53]. In the test phase, mice received repeated alcohol treatment spent more time to contact with the location unchanged object, which implied the impairment of recognition ability (see Fig. 1c). Rg1 pretreatment significantly ameliorated RAE induced deficit in recognition ability. These data demonstrated that repeated alcohol administration resulted in cognitive deficits in spatial learning and memory, and recognition ability could be effectively reversed by Rg1 treatment. All ginsenosides including Rg1 share the same dammaranetype triterpenoid structural [54]. Abundant evidence has demonstrated the neuroprotective effects of the 20(S)protopanaxadiol analogues [55]. Based on our finding, it can be inferred that the 20(S)-protopanaxadiol analogues may possess a similar potential effect on repeated alcohol 
induced psychomotor and cognitive deficits, though the precise mechanism remains to be further determined.

Chronic alcohol exposure leads to excessive release and accumulation of extracellular glutamate, a key excitatory neurotransmitter involved in vital physiological processes in the CNS $[19,21]$. Glutamate triggers over-activation and expression of calcium-permeable NMDARs, which leads to adaptive functional changes of these receptors [56-58]. Consequently, in the present research, repeated alcohol administration enhanced glutamate spillover and resulted in pathological changes of neurons in the CA1, CA3 and DG subregions of the hippocampus, which was possibly associated with the over-expression of NR2Bcontaining NMDARs. Most NMDARs are composed of two essential NR1 and two modulatory NR2 subunits [59]. The number of NMDAR is in a dynamic equilibrium between synaptic, extrasynaptic, and intracellular compartments [60]. The functional or pathological effects of NMDARs are closely related to their locations. Any shift in balance to enhance extrasynaptic NMDAR signaling may be detrimental to neuronal health. Synaptic NMDARs are thought to mediate neuronal plasticity, while extrasynaptic NMDARs are linked to $\mathrm{Ca}^{2+}$ regulation and glutamate excitotoxicity [61-63]. Excessive activation of extrasynaptic NMDARs initiates dysregulation of intracellular $\mathrm{Ca}^{2+}$ and enhances neuronal susceptibility to excitotoxic damage [64-66]. Activated NR2B-containing NMDARs subsequently leads to calpain activation [67]. Calpains are calcium-dependent proteases that have been implicated in a wide range of pathological states [68]. Distinct isoforms of calpains differentially activated by synaptic and extrasynaptic NMDAR stimulation, with the former activating calpain-1 ( $\mu$-calpain) and the latter activating calpain-2 (m-calpain) [69]. We further determined that repeated alcohol administration induced elevation of NR2B-containing NMDARs expression was associated with calpain-2 activation, which indicated that extrasynaptic NMDARs were excessively activated after RAE. Calpain-2, rather than calpain-1, results in proteolysis of downstream striatal-enriched protein phosphatase (STEP) and subsequent activation of p38 mitogen-activated protein kinase (p38 MAPK) [70].

STEP is highly enriched in the striatum, hippocampus, and cortex [71]. It forms a complex with the NMDAR and regulates the responsiveness of NMDAR to ethanol effects in the hippocampus [72, 73]. STEP cleaved from a membrane-associated STEP $_{61}$ isoform into a lower molecular-weight cytosolic-enriched $\mathrm{STEP}_{33}$ isoform during excitotoxic assault [74, 75]. In addition, p38 MAPK, which is expressed in extrasynaptic sites and

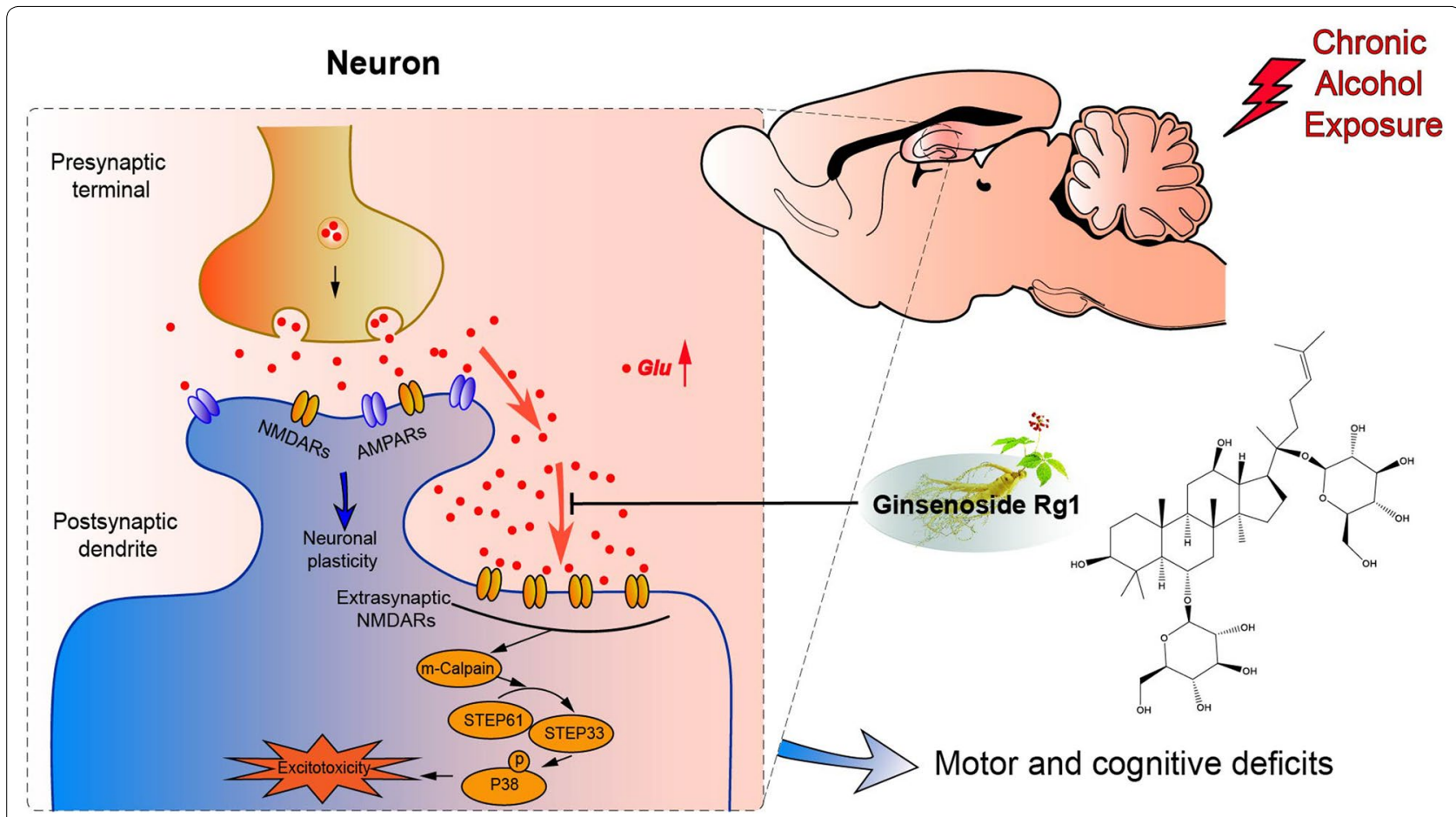

Fig. 5 A schematic diagram illustrating the proposed mechanism by which ginsenoside Rg1 alleviated repeated alcohol-induced cognitive deficits. Repeated alcohol exposure results in glutamate spillover and over-activation of extrasynaptic NR2B-containing NMDARs in the hippocampus. Activated extrasynaptic NMDARs initiates calpain-2/STEP/p38 excitotoxic cascade and leads to pathological changes of neurons in the hippocampus. Rg1 pretreatment can effectively ameliorate cognitive deficits via suppressing extrasynaptic NMDARs-mediated excitotoxic assaults 
implicated in NMDARs-mediated excitotoxic damage, is negatively regulated by STEP [70, 76]. Thus, reversing calpain-mediated STEP cleavage is sufficient to inhibit NMDARs-dependent p38 MAPK activation and protect neurons from excitotoxic damage. Intriguingly, we noticed that Rg1 treatment significantly reversed extrasynaptic NMDARs mediated excitotoxic signaling in a dose-dependent manner. Rg1 treatment suppressed the activation of calpain-2 and the transformation of STEP 61 to $\mathrm{STEP}_{33}$, which effectively inhibited the phosphorylation of p38 MAPK. Our findings suggested that repeated alcohol administration induced cognitive deficits could be attributed to NMDAR-mediated excitotoxic assault. Rg1 pretreatment significantly protected neurons in the hippocampus by suppressing excitotoxic NMDAR activity.

\section{Conclusion}

Data from the present research suggested that Rg1, at the optimal dose of $6 \mathrm{mg} / \mathrm{kg}$, has the potential to ameliorate excessive alcohol intake induced cognitive deficits by regulating extrasynaptic NMDARs-mediated excitotoxic signaling (breifly illustrated in Fig. 5). Our findings further provided a new strategy to treat chronic alcohol intoxication induced excitotoxicity and neurodegeneration.

\section{Abbreviations \\ CNS: Central nervous system; Glu: Glutamate; MWM: Morris water maze; NMDARs: N-methyl-D-aspartate receptors; OLR: Object location recognition; RAE: Repeated alcohol exposure; p38 MAPK: p38 mitogen-activated protein kinase; STEP: Striatal-enriched protein phosphatase.}

\section{Acknowledgements}

Not applicable.

\section{Authors' contributions}

$\mathrm{ZS}, \mathrm{DL}$ and $\mathrm{XL}$ designed the study and wrote the draft together; $\mathrm{LH}, \mathrm{ZP}, \mathrm{CL}$ and JL performed the study; $Y C, M Q$ analyzed the results. All authors read and approved the final manuscript.

\section{Funding}

This work was supported by the Scientific Research Fund of Hunan Provincial Education (19B422); China Postdoctoral Science Foundation (2019T120788, 2018M643369); Postdoctoral Fund of the First Affiliated Hospital, Jinan University (801324); the National Science and Technology Major Project (2017ZX09101002-002-008).

\section{Availability of data and materials \\ All the data used to support the findings of this study are available from the corresponding author upon reasonable request.}

Ethics approval and consent to participate Not applicable.

\section{Consent for publication}

Not applicable.

\section{Competing interests}

The authors declare that they have no competing interests.

\section{Author details}

${ }^{1}$ Division of Stem Cell Regulation and Application, Key Laboratory for Quality Evaluation of Bulk Herbs of Hunan Province, Hunan University of Chinese Medicine, Changsha 410208, Hunan, China. ${ }^{2}$ Guangdong-Hongkong-Macau Institute of CNS Regeneration, Ministry of Education CNS Regeneration Collaborative Joint Laboratory, Jinan University, Guangzhou 510632, China. ${ }^{3}$ Research Center for Pharmacology and Toxicology, Institute of Medicinal Plant Development (IMPLAD), Chinese Academy of Medical Sciences and Peking Union Medical College, Beijing 100193, China. ${ }^{4}$ Institute of Chinese Materia Medica, China Academy of Chinese Medical Sciences, Beijing 100700, China. ${ }^{5}$ College of Life Science and Technology, Beijing University of Chemical Technology, Beijing 100029, China.

Received: 23 February 2020 Accepted: 28 April 2020

Published online: 07 May 2020

\section{References}

1. Rehm J, et al. Global burden of disease and injury and economic cost attributable to alcohol use and alcohol-use disorders. Lancet. 2009;373(9682):2223-33.

2. Que J, Lu L, Shi L. Development and challenges of mental health in China. Gen Psychiatr. 2019;32(1):e100053.

3. Harper C. The neuropathology of alcohol-related brain damage. Alcohol Alcohol. 2009:44(2):136-40.

4. Mehta A, et al. Excitotoxicity: bridge to various triggers in neurodegenerative disorders. Eur J Pharmacol. 2013:698(1-3):6-18.

5. Maclin JMA, Wang T, Xiao S. Biomarkers for the diagnosis of Alzheimer's disease, dementia Lewy body, frontotemporal dementia and vascular dementia. Gen Psychiatr. 2019;32(1):e100054.

6. Oscar-Berman M, et al. Comparisons of Korsakoff and non-Korsakoff alcoholics on neuropsychological tests of prefrontal brain functioning. Alcohol Clin Exp Res. 2004;28(4):667-75.

7. Kopera M, et al. Cognitive functions in abstinent alcohol-dependent patients. Alcohol. 2012;46(7):665-71.

8. Chen J, et al. Clinical effectiveness of cognitive behavioural therapy on alcohol-dependent patients: an observation with the WeChat platform. Gen Psychiatr. 2019;32(5):e100087.

9. Sullivan EV, Pfefferbaum A. Neurocircuitry in alcoholism: a substrate of disruption and repair. Psychopharmacology. 2005;180(4):583-94.

10. Sanday $L$, et al. Ethanol-induced memory impairment in a discriminative avoidance task is state-dependent. Alcohol Clin Exp Res. 2013:37(Suppl 1):E30-9.

11. O'Daly OG, et al. Withdrawal-associated increases and decreases in functional neural connectivity associated with altered emotional regulation in alcoholism. Neuropsychopharmacology. 2012;37(10):2267-76.

12. Ozsoy S, Durak AC, Esel E. Hippocampal volumes and cognitive functions in adult alcoholic patients with adolescent-onset. Alcohol. 2013:47(1):9-14.

13. Chanraud $\mathrm{S}$, et al. Brain morphometry and cognitive performance in detoxified alcohol-dependents with preserved psychosocial functioning. Neuropsychopharmacology. 2007;32(2):429-38.

14. Shi Z, et al. Evaluation of an Abeta(1-40)-induced cognitive deficit in rat using a reward-directed instrumental learning task. Behav Brain Res. 2012;234(2):323-33.

15. Beresford TP, et al. Hippocampus volume loss due to chronic heavy drinking. Alcohol Clin Exp Res. 2006;30(11):1866-70.

16. Sullivan EV, et al. Longitudinal changes in cognition, gait, and balance in abstinent and relapsed alcoholic men: relationships to changes in brain structure. Neuropsychology. 2000;14(2):178-88.

17. Durazzo TC, et al. Cortical thickness, surface area, and volume of the brain reward system in alcohol dependence: relationships to relapse and extended abstinence. Alcohol Clin Exp Res. 2011;35(6):1187-200.

18. Staples MC, Kim A, Mandyam CD. Dendritic remodeling of hippocampal neurons is associated with altered NMDA receptor expression in alcohol dependent rats. Mol Cell Neurosci. 2015;65:153-62. 
19. Holmes A, Spanagel R, Krystal JH. Glutamatergic targets for new alcohol medications. Psychopharmacology. 2013;229(3):539-54.

20. Lovinger DM, White G, Weight FF. Ethanol inhibits NMDA-activated ion current in hippocampal neurons. Science. 1989;243(4899):1721-4.

21. Holmes A, et al. Chronic alcohol remodels prefrontal neurons and disrupts NMDAR-mediated fear extinction encoding. Nat Neurosci. 2012:15(10):1359-61.

22. Alfonso-Loeches S, Guerri C. Molecular and behavioral aspects of the actions of alcohol on the adult and developing brain. Crit Rev Clin Lab Sci. 2011:48(1):19-47.

23. Kumari M, Anji A. An old story with a new twist: do NMDAR1 mRNA binding proteins regulate expression of the NMDAR1 receptor in the presence of alcohol? Ann N Y Acad Sci. 2005:1053:311-8.

24. Pian JP, et al. $N$-methyl-D-aspartate receptor subunit expression in adult and adolescent brain following chronic ethanol exposure. Neuroscience. 2010;170(2):645-54

25. Fernandez-Moriano C, et al. Evaluation of the adaptogenic potential exerted by ginsenosides Rb1 and Rg1 against oxidative stressmediated neurotoxicity in an in vitro neuronal model. PLOS ONE. 2017;12(8):e0182933.

26. Wu J, et al. Ginsenoside Rg1 exerts a protective effect against Abeta(2)(5) $(-)(3)(5)$-induced toxicity in primary cultured rat cortical neurons through the NF-kappaB/NO pathway. Int J Mol Med. 2016;37(3):781-8.

27. Huang Y, Wu D, Fan W. Protection of ginsenoside Rg1 on chondrocyte from IL-1 beta-induced mitochondria-activated apoptosis through PI3K Akt signaling. Mol Cell Biochem. 2014;392(1-2):249-57.

28. Jiang $B$, et al. Antidepressant-like effects of ginsenoside $\mathrm{Rg} 1$ are due to activation of the BDNF signalling pathway and neurogenesis in the hippocampus. Br J Pharmacol. 2012;166(6):1872-87.

29. Liu Q, Kou JP, Yu BY. Ginsenoside Rg1 protects against hydrogen peroxide-induced cell death in PC12 cells via inhibiting NF-kappaB activation. Neurochem Int. 2011;58(1):119-25.

30. Dar NJ, et al. Attenuation of glutamate-induced excitotoxicity by Withanolide-A in neuron-like cells: role for PI3K/Akt/MAPK signalingpathway. Mol Neurobiol. 2018;55(4):2725-39.

31. Zhu G, et al. Chronic treatment with ginsenoside Rg1 promotes memory and hippocampal long-term potentiation in middle-aged mice. Neuroscience. 2015;292:81-9.

32. Song $X Y$, et al. Ginsenoside Rg1 attenuates okadaic acid induced spatial memory impairment by the GSK3beta/tau signaling pathway and the Abeta formation prevention in rats. Eur J Pharmacol. 2013;710(1-3):29-38.

33. Lu C, et al. Exploring the effect of ginsenoside Rh1 in a sleep deprivation-induced mouse memory impairment model. Phytother Res. 2017;31(5):763-70.

34. Zhang $Y$, et al. Post hoc power analysis: is it an informative and meaningful analysis? Gen Psychiatr. 2019;32(4):e100069.

35. Wang $B$, et al. The $p$-value and model specification in statistics. Gen Psychiatr. 2019:32(3):e100081.

36. Wang J, et al. Long-lasting adaptations of the NR2B-containing NMDA receptors in the dorsomedial striatum play a crucial role in alcohol consumption and relapse. J Neurosci. 2010;30(30):10187-98.

37. Kuzmin A, et al. Repeated moderate-dose ethanol bouts impair cognitive function in Wistar rats. Addict Biol. 2012;17(1):132-40.

38. Qin L, Crews FT. NADPH oxidase and reactive oxygen species contribute to alcohol-induced microglial activation and neurodegeneration. J Neuroinflamm. 2012;9:5.

39. Sullivan EV, Rosenbloom MJ, Pfefferbaum A. Pattern of motor and cognitive deficits in detoxified alcoholic men. Alcohol Clin Exp Res. 2000;24(5):611-21.

40. Bechara A. Decision making, impulse control and loss of willpower to resist drugs: a neurocognitive perspective. Nat Neurosci. 2005:8(11):1458-63.

41. Mechtcheriakov S, et al. A widespread distinct pattern of cerebral atrophy in patients with alcohol addiction revealed by voxel-based morphometry. J Neurol Neurosurg Psychiatry. 2007;78(6):610-4.

42. Koob GF, Volkow ND. Neurocircuitry of addiction. Neuropsychopharmacology. 2010;35(1):217-38.
43. Ferreira $V M$, et al. Ionotropic glutamate receptor subunit expression in the rat hippocampus: lack of an effect of a long-term ethanol exposure paradigm. Alcohol Clin Exp Res. 2001;25(10):1536-41.

44. Wu PH, et al. Correlated changes in NMDA receptor phosphorylation, functional activity, and sedation by chronic ethanol consumption. J Neurochem. 2010;115(5):1112-22.

45. Shi Z, et al. Fish oil treatment reduces chronic alcohol exposure induced synaptic changes. Addict Biol. 2019;24(4):577-89.

46. Morris R. Developments of a water-maze procedure for studying spatial learning in the rat. J Neurosci Methods. 1984;1 1(1):47-60.

47. Burgess N, Maguire EA, O'Keefe J. The human hippocampus and spatial and episodic memory. Neuron. 2002;35(4):625-41.

48. Dillon GM, et al. Excitotoxic lesions restricted to the dorsal CA1 field of the hippocampus impair spatial memory and extinction learning in C57BL/6 mice. Neurobiol Learn Mem. 2008;90(2):426-33.

49. Horner AJ, et al. A rapid, hippocampus-dependent, item-memory signal that initiates context memory in humans. Curr Biol. 2012;22(24):2369-74.

50. Barker GR, Warburton EC. When is the hippocampus involved in recognition memory? J Neurosci. 2011;31(29):10721-31.

51. Ennaceur A. One-trial object recognition in rats and mice: methodological and theoretical issues. Behav Brain Res. 2010;215(2):244-54.

52. Manns JR, Eichenbaum H. A cognitive map for object memory in the hippocampus. Learn Mem. 2009;16(10):616-24.

53. Lee I, Park SB. Perirhinal cortical inactivation impairs object-in-place memory and disrupts task-dependent firing in hippocampal CA1, but not in CA3. Front Neural Circuits. 2013;7:134.

54. Leung KW, et al. Angiomodulatory and neurological effects of ginsenosides. Curr Med Chem. 2007;14(12):1371-80.

55. Wu X, et al. Dammarane sapogenins ameliorates neurocognitive functional impairment induced by simulated long-duration spaceflight. Front Pharmacol. 2017:8:315.

56. Roberto $\mathrm{M}$, et al. Acute and chronic ethanol alter glutamatergic transmission in rat central amygdala: an in vitro and in vivo analysis. J Neurosci. 2004;24(7):1594-603.

57. Roberto M, et al. Chronic ethanol exposure and protracted abstinence alter NMDA receptors in central amygdala. Neuropsychopharmacology. 2006;31(5):988-96.

58. Lack AK, et al. Chronic ethanol and withdrawal differentially modulate pre- and postsynaptic function at glutamatergic synapses in rat basolateral amygdala. J Neurophysiol. 2007;98(6):3185-96.

59. Furukawa $\mathrm{H}$, et al. Subunit arrangement and function in NMDA receptors. Nature. 2005:438(7065):185-92.

60. Choquet D, Triller A. The dynamic synapse. Neuron. 2013;80(3):691-703.

61. Okamoto $S$, et al. Balance between synaptic versus extrasynaptic NMDA receptor activity influences inclusions and neurotoxicity of mutant huntingtin. Nat Med. 2009;15(12):1407-13.

62. Kaufman AM, et al. Opposing roles of synaptic and extrasynaptic NMDA receptor signaling in cocultured striatal and cortical neurons. J Neurosci. 2012;32(12):3992-4003.

63. Hardingham GE, Bading H. Synaptic versus extrasynaptic NMDA receptor signalling: implications for neurodegenerative disorders. Nat Rev Neurosci. 2010;11(10):682-96.

64. Parsons MP, Raymond LA. Extrasynaptic NMDA receptor involvement in central nervous system disorders. Neuron. 2014;82(2):279-93.

65. Benarroch EE. NMDA receptors: recent insights and clinical correlations. Neurology. 2011;76(20):1750-7.

66. Stark DT, Bazan NG. Synaptic and extrasynaptic NMDA receptors differentially modulate neuronal cyclooxygenase-2 function, lipid peroxidation, and neuroprotection. J Neurosci. 2011;31(39):13710-21.

67. Zhou M, Baudry M. Developmental changes in NMDA neurotoxicity reflect developmental changes in subunit composition of NMDA receptors. J Neurosci. 2006:26(11):2956-63.

68. Goll DE, et al. The calpain system. Physiol Rev. 2003;83(3):731-801

69. Wang $\mathrm{Y}$, et al. Distinct roles for mu-calpain and $\mathrm{m}$-calpain in synaptic NMDAR-mediated neuroprotection and extrasynaptic NMDAR-mediated neurodegeneration. J Neurosci. 2013;33(48):18880-92.

70. Xu J, et al. Extrasynaptic NMDA receptors couple preferentially to excitotoxicity via calpain-mediated cleavage of STEP. J Neurosci. 2009;29(29):9330-43. 
71. Lombroso PJ, et al. A protein tyrosine phosphatase expressed within dopaminoceptive neurons of the basal ganglia and related structures. J Neurosci. 1993;13(7):3064-74.

72. Pelkey KA, et al. Tyrosine phosphatase STEP is a tonic brake on induction of long-term potentiation. Neuron. 2002;34(1):127-38.

73. Hicklin TR, et al. Alcohol inhibition of the NMDA receptor function, longterm potentiation, and fear learning requires striatal-enriched protein tyrosine phosphatase. Proc Natl Acad Sci USA. 2011;108(16):6650-5.

74. Poddar R, et al. NR2B-NMDA receptor mediated modulation of the tyrosine phosphatase STEP regulates glutamate induced neuronal cell death. J Neurochem. 2010;115(6):1350-62
75. Braithwaite SP, et al. Expression and function of striatal enriched protein tyrosine phosphatase is profoundly altered in cerebral ischemia. Eur J Neurosci. 2008;27(9):2444-52.

76. Soriano FX, et al. Specific targeting of pro-death NMDA receptor signals with differing reliance on the NR2B PDZ ligand. J Neurosci. 2008;28(42):10696-710.

\section{Publisher's Note}

Springer Nature remains neutral with regard to jurisdictional claims in published maps and institutional affiliations.
Ready to submit your research? Choose BMC and benefit from:

- fast, convenient online submission

- thorough peer review by experienced researchers in your field

- rapid publication on acceptance

- support for research data, including large and complex data types

- gold Open Access which fosters wider collaboration and increased citations

- maximum visibility for your research: over $100 \mathrm{M}$ website views per year

At BMC, research is always in progress.

Learn more biomedcentral.com/submissions 